\title{
Evaluación de Riesgo por Estrés Térmico en Trabajadores de los Procesos de Incineración y Secado de una Empresa de Tableros Contrachapados
}

\author{
Raúl E. Gutiérrez ${ }^{(1)}$, Karla B. Guerra ${ }^{(1)}$, Marco D. Gutiérrez ${ }^{(2)}$ \\ (1) Escuela Superior Politécnica de Chimborazo, Panamericana Sur Km 11/2, Riobamba- Ecuador \\ (2) Arboriente S.A., Av Ceslao Marín y Cueva de los Tayos, Puyo- Ecuador \\ E-mail: neto1516@hotmail.com, karla._7@hotmail.com, dgutierrez@arboriente.com.ec
}

Recibido Sep. 6, 2017; Aceptado Nov. 21, 2017; Versión final Ene. 21, 2018, Publicado Jun. 2018

\begin{abstract}
Resumen
Esta investigación tiene como objetivo evaluar el riesgo laboral por estrés térmico en los trabajadores de los procesos de incineración y secado en la empresa Arboriente S.A. Se trata de un estudio de campo, de tipo descriptivo y que se encuentra enmarcado dentro de la investigación cuantitativa. La población estuvo integrada por veinte trabajadores de los procesos mencionados y para la evaluación del riesgo se utilizó el método del índice Wet Bulb Globe Temperature (WBGT), el cual se basa en la caracterización de los puestos de trabajo, la medición de las temperaturas de bulbo húmedo, bulbo seco y de globo, la determinación de la carga térmica metabólica y el cálculo y valoración del índice. Como resultado se obtuvo que en ninguno de los puestos de trabajo evaluados existe riesgo higiénico por estrés térmico, debido principalmente a la suficiente aclimatación de los trabajadores y a su baja carga térmica metabólica.
\end{abstract}

Palabras clave: estrés térmico; riesgo laboral; riesgo higiénico; índice WBGT; carga térmica metabólica

\section{Thermal Stress Risk Assessment on Workers in the Incineration and Drying Processes of a Plywood Company}

\begin{abstract}
The objective of this research is to evaluate the occupational risk due to thermal stress on workers in the incineration and drying processes at Arboriente S.A. The research characteristics consist of a field study, which is descriptive and quantitative. The population included twenty workers who perform the stated processes. For risk assessment, the Wet Bulb Globe Temperature Index (WBGT) was used. It is based on the characterization of the workstations, the measurement of the temperatures of Wet bulb, dry bulb and globe. In addition, the determination of the metabolic thermal load, and the calculation and evaluation of the index are also included. As a result, no hygienic risk was evidenced on any of the evaluated workstations attributable to thermal stress. This is mainly due to sufficient acclimatization of the workers and their low metabolic thermal load.
\end{abstract}




\section{INTRODUCCIÓN}

Según la American Industrial Hygiene Association (AlHA) la higiene industrial es la "ciencia y arte dedicados al reconocimiento, evaluación y control de aquellos factores ambientales o tensiones emanadas o provocadas por el lugar de trabajo y que pueden ocasionar enfermedades, destruir la salud y el bienestar o crear algún malestar significativo entre los trabajadores o los ciudadanos de una comunidad" (Falagán et al., 2000). El calor es uno de los contaminantes físicos más comunes en los ambientes laborales, que puede generar entre los trabajadores expuestos desde sensaciones de pérdida del confort, hasta afecciones de mayor gravedad con el potencial de comprometer su salud, lo cual reduce el rendimiento laboral (Fisk, 2000; Wyon, 2004).

La temperatura corporal es una constante biológica que en condiciones normales oscila entre 35,8 y $37,2{ }^{\circ} \mathrm{C}$ (Pérez et al., 2004) El estrés por calor es el disconfort y tensión fisiológica que se ocasiona por la exposición a ambientes calurosos, principalmente durante el trabajo físico (Piñeiro et al., 2004) y es un factor importante en situaciones industriales, eventos deportivos y escenarios militares. (Epstein y Moran, 2006)

Los trabajadores que desarrollan sus labores en ambientes calurosos y realizan un esfuerzo físico significativo pueden ver afectado su sistema de regulación térmica corporal (Camacho, 2013), impidiendo un intercambio normal de temperatura entre su cuerpo y el ambiente que les rodea, esto permite que el calor se acumule en el organismo y pueda llegar a niveles en que los daños producidos sean irreversibles (INSHT, 1992).

El estrés por calor es un problema conocido especialmente en países tropicales que afecta a la salud y el bienestar de los trabajadores. En un estudio realizado en Thailandia se ha identificado que trabajar bajo condiciones de estrés calórico se asocia con una peor salud general y malestar psicológico, (Tawatsupa et al., 2010) además puede disminuir la tolerancia a otros peligros ambientales. (Epstein y Moran, 2006).

En ocasiones puede existir la progresión de estrés por calor a golpe de calor, que es una emergencia médica caracterizada por un incremento de la temperatura corporal central por encima de $40^{\circ} \mathrm{C}$. (Curley e Irwin, 2003) El golpe de calor ocasiona alteraciones en el sistema termorregulador, en la expresión de proteínas de shock térmico y en el sistema nervioso central, lo que causa encefalopatía, coma y si no se toman las medidas oportunas, puede provocar la muerte. (Piñeiro et al., 2004) El estrés por calor ocupacional afectó a casi el $20 \%$ de los trabajadores tailandeses en la cohorte nacional. (Tawatsupa et al., 2013).

Según la Administración de Seguridad y Salud Laboral OSHA (2013), en los Estados Unidos entre 2003 y 2012, alrededor de 30 trabajadores murieron anualmente debido a enfermedades y lesiones relacionadas con el calor. (Yi y Chan, 2017) Estudios realizados en Sudáfrica han informado muertes relacionadas con el calor en el trabajo debido a la hipertermia o golpe de calor (Wyndham, 1965). Además en los Estados Unidos un estudio ha analizado 423 muertes que se relacionan con estrés térmico en trabajadores agrícolas (MMWR, 2008).

El estrés laboral no solo afecta al individuo, sino también a la productividad en el trabajo, el rendimiento y la economía local. (Kjellstrom et al., 2016a, 2017b) Puesto que el aumento de la temperatura reduce la productividad en el trabajo, la productividad económica mundial podría disminuir y afectar de manera desproporcionada a países en la zona climática tropical. (Lundgren et al., 2013). Dunne et al. (2013) han estimado que el estrés por calor ambiental ha reducido significativamente la capacidad laboral mundial en los meses pico y han previsto una reducción adicional del $80 \%$ para 2050. Zander et al. (2015) han estimado que la productividad laboral podría reducir en un 11-27\% para fines de este siglo en áreas cálidas (Yi y Chan, 2017).

El calentamiento global está generando olas de calor más frecuentes y severas generando eventos de alto impacto como la Sequía en África entre 2010- 2012 lo que dio como resultado más de 258.000 muertes y olas de calor en Pakistán e India en 2015, que causaron alrededor de 4.100 muertes, esto según la Organización Meteorológica Mundial (WMO, 2015) (Yi y Chan, 2017). Las personas con enfermedades cardíacas y cardiovasculares son más vulnerables y tienen un mayor riesgo de enfermedad debido al calor relacionado con el calentamiento global. (Yi y Chan, 2015; Vandentorren et al., 2016)

En la evaluación de los posibles riesgos laborales derivados de la exposición al calor, intervienen una serie de variables a considerar, entre las que se incluyen, la existencia de fuentes de irradiación, elevados porcentajes de humedad y temperatura ambiente, condiciones corporales específicas de cada trabajador, vestimenta y la carga de exigencias físicas en las tareas realizadas (Ramsey, 1978).

En 1957, mientras se desarrollaban varios índices de estrés por calor, Yaglou y Minard introdujeron el índice Wet Bulb Globe Temperature (WBGT) o índice de temperatura de globo de bulbo húmedo, el cual se determina mediante tres parámetros: temperatura de globo, temperatura de bulbo húmedo y temperatura de bulbo seco (Moran y Pandolf, 1999). Luego de varias mejoras fue adaptado por el Instituto Nacional para la 
Seguridad y Salud Ocupacional de los Estados Unidos (NIOSH, 1972) y posteriormente por ISO (1989) como estándar internacional. (Oliveira et al, 2015)

El índice WBGT después de más de 50 años de uso sigue considerándose un índice ampliamente utilizado, se aplica en varios campos como el industrial, deportivo, militar, entre otros, y se ha incluido en normas nacionales, regionales e internacionales (Budd, 2007; Parsons, 2006). Es el índice de estrés por calor más comúnmente utilizado en todo el mundo (p. ej., se utiliza en Australia, China, India, Japón, Reino Unido, Estados Unidos y la Unión Europea) (Yang et al., 2017). Este índice se convirtió en el más común para describir el estrés térmico ambiental debido a su simplicidad y aplicabilidad. Es utilizado por el ejército de los Estados Unidos de América y por las asociaciones deportivas para establecer directrices de seguridad de los entrenamientos para prevenir lesiones por exposición al calor. (Montain et al., 1999; Armstrong et al., 1996)

El marco normativo en vigor en el Ecuador en el Decreto Ejecutivo 2393 de 1986 establece la obligatoriedad de los empleadores de adoptar las medidas necesarias para la prevención de los riesgos que puedan afectar a la salud y al bienestar de los trabajadores en los lugares de trabajo de su responsabilidad. Del mismo modo la legislación nacional vigente establece que en la evaluación de los ambientes térmicos y del riesgo laboral por estrés térmico, se aceptará la metodología del índice WBGT y se asumirán los límites permisibles establecidos en la norma ISO 7243 de 1989. En este sentido se realizó un proceso de medición de las variables térmicas de los ambientes de trabajo de los procesos de incineración y secado de Arboriente S.A., empresa Ecuatoriana dedicada a la producción y comercialización de tableros de madera contrachapada, en la que la energía necesaria en secaderos y prensas proviene de la combustión de biomasa (residuos maderables). En este trabajo se usa el método del índice WBGT para evaluar la existencia o no de riesgo por estrés térmico en los trabajadores de los puestos de trabajo afectados y proponer las medidas de control necesarias.

\section{METODOLOGÍA}

El presente estudio es de tipo descriptivo y se enmarca dentro de la investigación cuantitativa, este tipo de estudio busca identificar características específicas de sujetos, procesos o fenómenos a estudiar, en ellos a través de la recolección y evaluación de la información sobre los parámetros que componen el fenómeno de análisis, se pueden establecer conclusiones para las condiciones específicas del estudio (Hernández et al., 2006). Se ha escogido este tipo de estudio debido a que permite calcular y analizar las variables de temperatura, humedad y carga térmica metabólica que en conjunto determinan el nivel de riesgo higiénico por estrés térmico en los puestos de trabajo analizados.

\section{Población y muestra}

La población está formada por los operadores de la maquinaria (20 trabajadores) de los procesos de incineración de biomasa y secado de chapas de madera de la empresa. Debido a que la población se encuentra distribuida en dos turnos de trabajo, se definió como muestra del estudio a 10 trabajadores que desarrollan sus labores en el primer turno de trabajo (06:00 - 14:00) y representan el 50\% de la población expuesta.

\section{Recolección de la Información}

La recopilación de la información necesaria tanto para la caracterización de los puestos de trabajo como la de los parámetros ambientales medidos en sitio, se realizó a través de formatos previamente elaborados.

La información de los parámetros ambientales fue analizada a través de las fórmulas correspondientes al método del índice WBGT, mientras que los datos sobre la carga física de las tareas identificadas fueron analizados a través de tablas referenciales para el cálculo de la carga térmica metabólica.

\section{Instrumentos de Medición}

En la medición de parámetros ambientales como el porcentaje de humedad, las temperaturas de bulbo húmedo, bulbo seco y de globo, se utilizó un instrumento medidor de estrés térmico debidamente calibrado, de marca General, modelo WBGT 8778. En la medición de parámetros como la velocidad y flujo de aire, se utilizó un anemómetro debidamente calibrado, de marca PYLE, modelo PMA82.

\section{Descripción del Método}

Las etapas en las cuales se desarrolla este estudio se muestran en la figura 1. Para la evaluación del riesgo por estrés térmico se utilizó el método del índice WBGT, este se basa en la medición de variables ambientales presentes en los locales de trabajo (Cújar y Julio, 2016). Con base en los resultados obtenidos en la medición de los parámetros de temperatura se realiza el cálculo del índice WBGT para cada una de las tareas analizadas y en dependencia del tipo de trabajo (interior o exterior), el método establece dos ecuaciones específicas. 


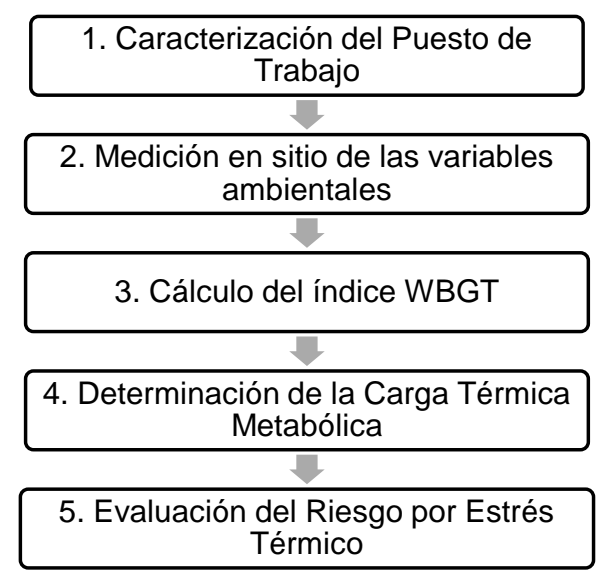

Fig. 1: Método del Índice WBGT

El cálculo del índice WBGT en trabajos de interiores se presenta en la ecuación (1), mientras que el cálculo para trabajos en exteriores se presenta en la ecuación (2), donde THN es la temperatura de bulbo húmedo, TG es la temperatura de globo y TA es la temperatura ambiente.

WBGT $=0,7 T H N+0,3 T G$

WBGT $=0,7 T H N+0,2 T G+0,1 T A$

A partir de los valores de la duración de las tareas en minutos por hora y de los resultados del índice WBGT obtenidos para cada una de ellas, se calcula el índice WBGT medio, valor ponderado para cada hora de trabajo que se obtiene a través de la ecuación (3), donde WBGT es el índice calculado para cada tarea y $t$ es su tiempo de duración en minutos.

WBGT $=\frac{\sum_{i=1}^{n}\left(W_{B G T} \times t_{i}\right)}{\sum_{i=1}^{n} t_{i}}$

Se procede con la determinación de la Carga Térmica Metabólica (CTM), valor que representa la energía expresada en Kilocalorías por minuto que consumen los trabajadores en la ejecución de cada una de sus tareas. Este valor se define tomando como referencia la información de las tablas 1 y 2.

Tabla 1: Posición y movimiento del cuerpo. (Tomado, con autorización, de INSHT, 1993)

\begin{tabular}{|l|l|}
\hline Posición y movimiento del cuerpo & Kcal/ Min \\
\hline Sentado & 0,3 \\
\hline De pié & 0,6 \\
\hline Andando & $2,0-3,0$ \\
\hline Subida de una pendiente andando & añadir 0,8 por $m$ de subida \\
\hline
\end{tabular}

Tabla 2: Tipo de Trabajo. (Tomado, con autorización, de INSHT, 1993)

\begin{tabular}{|c|c|c|c|}
\hline \multicolumn{2}{|l|}{ Tipo de trabajo } & Valor medio (Kcal/min) & Rango (Kcal/min) \\
\hline \multirow[b]{2}{*}{ Trabajo manual } & Ligero & 0,4 & \multirow{2}{*}{$0,2-1,2$} \\
\hline & Pesado & 0,9 & \\
\hline \multirow[b]{2}{*}{ Trabajo con un brazo } & Ligero & 1,0 & \multirow{2}{*}{$0,7-2,5$} \\
\hline & Pesado & 1,7 & \\
\hline \multirow[b]{2}{*}{ Trabajo con dos brazos } & Ligero & 1,5 & \multirow{2}{*}{$1,0-3,5$} \\
\hline & Pesado & 2,5 & \\
\hline \multirow{4}{*}{ Trabajo con el cuerpo } & Ligero & 3,5 & \multirow{4}{*}{$2,5-15,0$} \\
\hline & Moderado & 5,0 & \\
\hline & Pesado & 7,0 & \\
\hline & Muy pesado & 9,0 & \\
\hline
\end{tabular}


A partir de los valores de la duración de las tareas en minutos por hora y de los resultados de la CTM obtenidos para cada una de ellas, se calcula la CTM media, valor ponderado para cada hora de trabajo que se obtiene a través de la ecuación (4) donde CTM es la Carga Térmica Metabólica calculada para cada tarea y $t$ es su tiempo de duración en minutos.

$\mathrm{CTM}=\frac{\sum_{\mathrm{i}=1}^{\mathrm{n}}\left(\left(\mathrm{CTM} \times \mathrm{t}_{\mathrm{i}}\right)\right.}{\sum_{\mathrm{i}=1}^{\mathrm{n}} \mathrm{t}_{\mathrm{i}} \times 60}$

Con base en el valor obtenido de la CTM para cada hora de trabajo se determina el WGBT límite, valor de referencia expresado en ${ }^{\circ} \mathrm{C}$ que permite la valoración del riesgo y que se obtiene a partir de la información de la tabla 3.

Tabla 3: Determinación del WBGT límite. (Tomado, con autorización, de INSHT, 1993)

\begin{tabular}{|c|c|c|c|c|}
\hline \multirow{3}{*}{$\begin{array}{c}\text { Calor metabólico } \\
\mathrm{Kcal} / \mathrm{h}\end{array}$} & \multicolumn{4}{|c|}{ WBGT límite ${ }^{\circ} \mathrm{C}$} \\
\hline & \multicolumn{2}{|c|}{ Persona aclimatada } & \multicolumn{2}{|c|}{ Persona no aclimatada } \\
\hline & $V=0$ & $V \neq 0$ & $V=0$ & $V \neq 0$ \\
\hline$\leq 100$ & 33 & 33 & 32 & 32 \\
\hline $100-200$ & 30 & 30 & 29 & 29 \\
\hline 200-310 & 28 & 28 & 26 & 26 \\
\hline $310-400$ & 25 & 26 & 22 & 23 \\
\hline$>400$ & 23 & 25 & 18 & 20 \\
\hline
\end{tabular}

Para la valoración del riesgo se realiza una división entre los valores del WBGT medio y el WBGT límite, si el valor resultante es igual o mayor a 1 indica que existe riesgo higiénico por estrés térmico y si el valor resultante es menor a 1 se concluye que no existe dicho riesgo.

\section{RESULTADOS Y DISCUSIÓN}

Como se indicó antes, el presente estudio se desarrolló en las instalaciones de Arboriente S.A., empresa Ecuatoriana dedicada a la producción y comercialización de tableros de madera contrachapada. En el proceso de incineración se realiza la combustión de biomasa (residuos maderables) con la finalidad de generar vapor saturado a altas temperaturas, necesario para el funcionamiento de secaderos y prensa en caliente. Las principales fuentes de irradiación de calor son la caldera de combustión y las instalaciones que permiten la distribución del vapor saturado.

El proceso de secado se basa en el paso de las láminas de madera por una serie de cámaras, en las cuales a partir del vapor saturado y por medio de la circulación de aire caliente, se busca reducir el porcentaje de humedad del material. Las principales fuentes de irradiación de calor en este proceso son: las cámaras de los dos equipos de secado y las instalaciones que permiten la distribución del vapor saturado. Además de las fuentes de irradiación de calor descritas, es importante mencionar que la temperatura promedio anual y la humedad relativa media en la ciudad del Puyo se sitúan en torno a los $21,2^{\circ} \mathrm{C}$ y al $88 \%$ respectivamente (INHAMI, 2012), la climatización de las instalaciones se realiza a través de sistemas eólicos de acción mecánica, distribuidos a lo largo de la planta de producción para la continua renovación de aire.

\section{Caracterización de los puestos de trabajo}

Los procesos de incineración y secado se desarrollan en dos turnos de trabajo: el primero de 06:00 a 14:00 y el segundo de 14:00 a 22:00, en ellos laboran un total de 10 trabajadores por turno (20 en total), los cuales ejecutan principalmente tareas de operación de maquinaria, abastecimiento de combustible, alimentación, recepción y verificación de material en proceso (láminas de madera).

A través de la observación de las tareas y el análisis de la organización y condiciones del trabajo, se han identificado 4 puestos de trabajo correspondientes a los procesos de análisis, en el proceso de secado, los puestos de "Operador de Secadero de Caras" y "Operador de Secadero de Intermedios" que cuentan con 3 trabajadores por turno respectivamente. En el proceso de incineración, los puestos de "Operador de Trituradora" y "Operador de Caldera" cada uno con 2 trabajadores por turno. Las características principales de los puestos de trabajo se presentan en la tabla 4. 
Tabla 4: Caracterización de los puestos de trabajo

\begin{tabular}{|c|c|c|c|c|c|c|}
\hline Proceso & $N^{\circ}$ & Puesto de trabajo & $N^{\circ}$ & Descripción de la Tarea & $\begin{array}{l}\text { Lugar de } \\
\text { la tarea }\end{array}$ & $\begin{array}{c}\text { Tiempo de } \\
\text { duración }(\mathrm{min} / \mathrm{h})\end{array}$ \\
\hline \multirow{6}{*}{$\begin{array}{l}\text { D } \\
\text { ర్ } \\
\infty\end{array}$} & \multirow{3}{*}{1} & \multirow{3}{*}{$\begin{array}{l}\text { Operador } \\
\text { Secadero de } \\
\text { Caras }\end{array}$} & 1 & Abastecer de material húmedo & Interior & 20 \\
\hline & & & 2 & Apilar Material Seco & Interior & 20 \\
\hline & & & 3 & Esperar paso de material & Interior & 20 \\
\hline & \multirow{3}{*}{2} & \multirow{3}{*}{$\begin{array}{l}\text { Operador } \\
\text { Secadero de } \\
\text { Intermedios }\end{array}$} & 1 & Abastecer de material húmedo & Interior & 20 \\
\hline & & & 2 & Apilar Material Seco & Interior & 20 \\
\hline & & & 3 & Esperar paso de material & Interior & 20 \\
\hline \multirow{7}{*}{$\begin{array}{l}\frac{5}{0} \\
\frac{0}{0} \\
\frac{0}{0} \\
: \frac{5}{0} \\
\text { S }\end{array}$} & \multirow{3}{*}{1} & \multirow{3}{*}{$\begin{array}{l}\text { Operador } \\
\text { Trituradora }\end{array}$} & 1 & $\begin{array}{l}\text { Descargar banda transportadora de } \\
\text { desperdicios }\end{array}$ & Interior & 20 \\
\hline & & & 2 & Transporte manual de coches & Interior & 10 \\
\hline & & & 3 & $\begin{array}{l}\text { Abastecer de material de choches en } \\
\text { banda trituradora }\end{array}$ & Interior & 30 \\
\hline & \multirow{4}{*}{2} & \multirow{4}{*}{$\begin{array}{l}\text { Operador } \\
\text { Caldera }\end{array}$} & 1 & $\begin{array}{l}\text { Verificación y operación tablero de } \\
\text { control caldera }\end{array}$ & Interior & 40 \\
\hline & & & 2 & Ayuda en chipeadora & Interior & 10 \\
\hline & & & 3 & Alimentación manual de caldera & Interior & 7 \\
\hline & & & 4 & Desalojo de ceniza & Exterior & 3 \\
\hline
\end{tabular}

\section{Medición en sitio de variables ambientales}

El proceso de medición se desarrolló en dos días que correspondieron a la tercera semana del mes de mayo de 2017, en los cuales durante el primer turno de trabajo y asegurando condiciones normales de operación y funcionamiento de la maquinaria, se visitaron las instalaciones en donde se desarrollan las tareas de los puestos de trabajo analizados.

Para cada una de las principales tareas que supongan un riesgo de estrés térmico por exposición a calor, se midieron tres parámetros ambientales: temperatura de bulbo húmedo (THN), temperatura de globo (TG) y temperatura de bulbo seco o ambiente (TA); se realizaron mediciones en tres diferentes posiciones corporales, a nivel de los tobillos, a nivel del abdomen y a nivel de la cabeza; estos valores se promediaron para obtener el resultado de cada uno de los parámetros, los cuales se incorporarán en la ecuación para el cálculo del índice WBGT tanto para tareas en exteriores como en interiores. Los valores obtenidos en las mediciones, así como el cálculo del índice WBGT por tarea se presentan en la tabla 5.

Tabla 5: Medición de variables ambientales

\begin{tabular}{|c|c|c|c|c|c|c|c|}
\hline \multirow[b]{2}{*}{$\begin{array}{l}N^{\circ} \text { Puesto } \\
\text { de trabajo }\end{array}$} & \multirow[b]{2}{*}{$N^{\circ}$ Tarea } & \multicolumn{6}{|c|}{ Mediciones } \\
\hline & & $\begin{array}{c}T \text { de bulbo } \\
\text { húmedo }\left({ }^{\circ} \mathrm{C}\right)\end{array}$ & $\begin{array}{c}\text { T de globo } \\
\left({ }^{\circ} \mathrm{C}\right)\end{array}$ & $\begin{array}{c}T \text { ambiental } \\
\left({ }^{\circ} \mathrm{C}\right)\end{array}$ & $\begin{array}{l}\text { Vel. viento } \\
(\mathrm{m} / \mathrm{s})\end{array}$ & $\begin{array}{c}\text { WBGT } \\
\text { interior } \\
\left({ }^{\circ} \mathrm{C}\right)\end{array}$ & $\begin{array}{c}\text { WBGT } \\
\text { exterior }\left({ }^{\circ} \mathrm{C}\right)\end{array}$ \\
\hline \multirow{3}{*}{1} & 1 & 22,67 & 28,1 & 27,7 & 0 & 24,3 & \\
\hline & 2 & 23,05 & 29,2 & 28,1 & 0 & 24,9 & \\
\hline & 3 & 22,87 & 29,3 & 28,6 & 0 & 24,8 & \\
\hline \multirow{3}{*}{2} & 1 & 22,66 & 29,8 & 28,8 & 0 & 24,8 & \\
\hline & 2 & 22,09 & 29,8 & 28,2 & 0 & 24,4 & \\
\hline & 3 & 22,11 & 29,4 & 28,1 & 0 & 24,3 & \\
\hline \multirow{3}{*}{3} & 1 & 22,81 & 29,1 & 28,6 & 0 & 24,7 & \\
\hline & 2 & 22,2 & 29,2 & 28,9 & 0 & 24,3 & \\
\hline & 3 & 22,37 & 28,8 & 28,2 & 0 & 24,3 & \\
\hline \multirow{4}{*}{4} & 1 & 22,42 & 29 & 25,3 & 0 & 24,4 & \\
\hline & 2 & 22,54 & 29,4 & 24,9 & 0 & 24,6 & \\
\hline & 3 & 23,02 & 30,9 & 27,9 & 0 & 25,4 & \\
\hline & 4 & 23,86 & 27,3 & 28,4 & 0 & & 25,0 \\
\hline
\end{tabular}




\section{Cálculo del índice WBGT medio}

A partir de los valores obtenidos en el proceso de medición y los índices WBGT por tarea, se calculó el valor del índice WBGT medio expresado en ${ }^{\circ} \mathrm{C}$, para lo cual se utilizó la ecuación (3). Los resultados por puesto de trabajo se presentan en la tabla 6.

\section{Determinación de la carga térmica metabólica}

Con base en el análisis y caracterización de las tareas y utilizando las tablas de referencia 1 y 2, se determinó la CTM por tarea expresada en Kilocalorías por minuto. A partir de los resultados y utilizando la ecuación (4) se calculó la CTM media por puesto de trabajo expresada en Kilocalorías por hora, los resultados se muestran en la tabla 6, en esta se observa que los índices WBGT medio de cada uno de los puestos de trabajo analizados se encuentran en torno a los $24^{\circ} \mathrm{C}$, siendo el mayor, el correspondiente al puesto: "Operador de Secadero de Caras" con un valor de $24,66^{\circ} \mathrm{C}$.

Tabla 6: WBGT medio y CTM media

\begin{tabular}{|c|c|c|c|c|c|c|c|}
\hline \multirow[b]{2}{*}{$\begin{array}{l}N^{\circ} \text { Puesto } \\
\text { de trabajo }\end{array}$} & \multirow[b]{2}{*}{$\begin{array}{c}\mathrm{N}^{\circ} \\
\text { Tarea }\end{array}$} & \multicolumn{6}{|c|}{ Cálculos } \\
\hline & & $\begin{array}{l}\text { WBGT } \\
\text { medio } \\
\left({ }^{\circ} \mathrm{C}\right)\end{array}$ & $\begin{array}{c}\text { Posición y } \\
\text { movimiento del } \\
\text { cuerpo (Kcal/ min) }\end{array}$ & $\begin{array}{c}\text { Tipo de } \\
\text { trabajo (Kcal/ } \\
\text { min) }\end{array}$ & $\begin{array}{c}\text { Metabolismo } \\
\text { basal (Kcal/ } \\
\text { min) }\end{array}$ & $\begin{array}{l}\text { Carga térmica } \\
\text { metabólica } \\
(\text { Kcal/ h) }\end{array}$ & $\begin{array}{c}\text { CTM } \\
\text { Media } \\
\text { (Kcal/h) }\end{array}$ \\
\hline \multirow{3}{*}{1} & 1 & \multirow{3}{*}{24,66} & 0,3 & 0,4 & 1 & 1,7 & \multirow{3}{*}{136} \\
\hline & 2 & & 0,6 & 0,4 & 1 & 2 & \\
\hline & 3 & & 0,6 & 1,5 & 1 & 3,1 & \\
\hline \multirow{3}{*}{2} & 1 & \multirow{3}{*}{24,50} & 0,6 & 0,9 & 1 & 2,5 & \multirow{3}{*}{134} \\
\hline & 2 & & 0,6 & 0,9 & 1 & 2,5 & \\
\hline & 3 & & 0,3 & 0,4 & 1 & 1,7 & \\
\hline \multirow{3}{*}{3} & 1 & \multirow{3}{*}{24,43} & 0,6 & 1,5 & 1 & 3,1 & \multirow{3}{*}{240} \\
\hline & 2 & & 2 & 2,5 & 1 & 5,5 & \\
\hline & 3 & & 0,6 & 2,5 & 1 & 4,1 & \\
\hline \multirow{4}{*}{4} & 1 & \multirow{4}{*}{24,57} & 0,3 & 0,4 & 1 & 1,7 & \multirow{4}{*}{140} \\
\hline & 2 & & 0,6 & 2,5 & 1 & 4,1 & \\
\hline & 3 & & 0,6 & 1,5 & 1 & 3,1 & \\
\hline & 4 & & 0,6 & 1,5 & 1 & 3,1 & \\
\hline
\end{tabular}

En cuanto a la CTM media derivada de las exigencias físicas de las tareas en cada puesto de trabajo, considera que el puesto con mayor gasto energético es el de "Operador de Trituradora" con 240 Kilocalorías por hora, mientras que el puesto con el menor desgaste es el de "Operador de Secadero de Intermedios" con 136 Kilocalorías por hora. Es importante mencionar que en todos los puestos analizados se verificó el desarrollo de un régimen de trabajo continuo durante las 8 horas del turno laboral. En la figura 2 se muestra la carga térmica metabólica por puesto de trabajo.

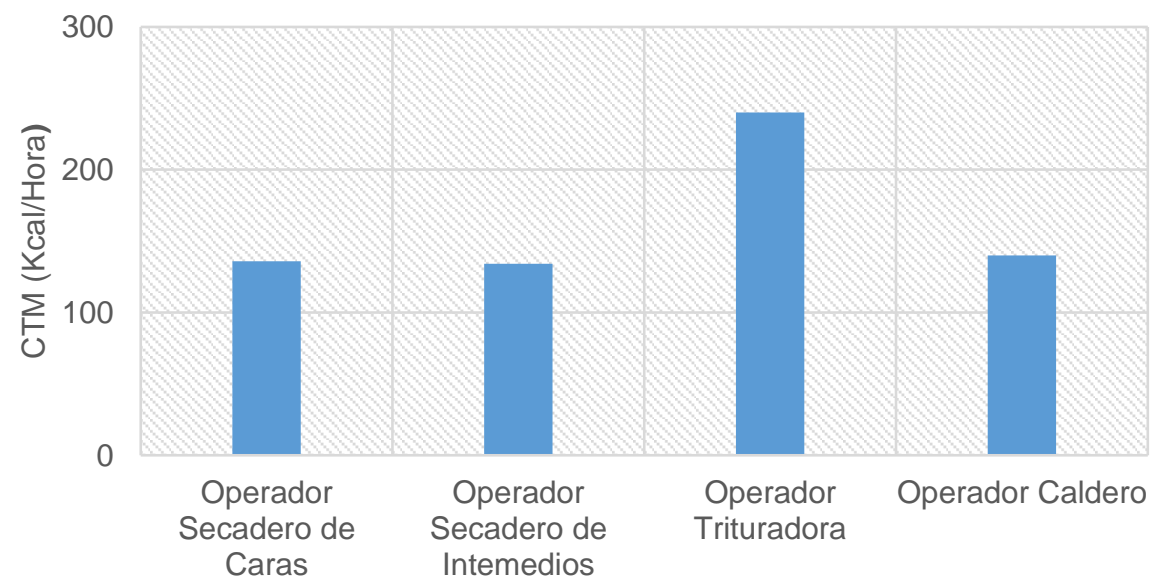

Fig. 2: CTM media por puesto de trabajo 


\section{Evaluación del riesgo por estrés térmico}

En el proceso de valoración del riesgo por estrés térmico a través del método del índice WBGT, es necesario determinar un valor WGBT de referencia, que constituirá el valor límite de exposición a un cierto nivel de temperatura (WBGT medio) para un puesto de trabajo con un desgaste energético previamente definido (CTM media). De este modo se procedió con la determinación de los valores del WBGT límite para cada uno de los puestos de trabajo analizados. Para ello se tomaron como punto de partida los valores CTM medio y utilizando como referencia los valores de la tabla 3 , se obtuvieron los resultados que se muestran en la tabla 7.

Tabla 7: Determinación del WBGT límite

\begin{tabular}{|c|c|c|c|c|}
\hline $\begin{array}{c}\mathrm{N}^{\circ} \text { Puesto de } \\
\text { trabajo }\end{array}$ & $\begin{array}{c}\text { Persona } \\
\text { aclimatada }\end{array}$ & $\begin{array}{c}\text { CTM media } \\
(\text { Kcal/ } \mathrm{h})\end{array}$ & $\begin{array}{c}\text { Velocidad del } \\
\text { viento }(\mathrm{m} / \mathrm{s})\end{array}$ & $\begin{array}{c}\text { WBGT límite } \\
\left({ }^{\circ} \mathrm{C}\right)\end{array}$ \\
\hline 1 & $\mathrm{SI}$ & 136 & 0 & 30 \\
\hline 2 & $\mathrm{SI}$ & 134 & 0 & 30 \\
\hline 3 & $\mathrm{SI}$ & 240 & 0 & 28 \\
\hline 4 & $\mathrm{SI}$ & 140 & 0 & 30 \\
\hline
\end{tabular}

En el desarrollo del estudio se verificó que la vestimenta utilizada por los trabajadores es en su totalidad ropa ligera, pantalones tipo jean, camiseta manga corta de algodón y calzado de seguridad, por lo tanto se consideró que este factor no afecta en el cálculo de los índices y en la determinación del WBGT límite (INSHT, 1992). Finalmente en la determinación de la existencia o no del riesgo higiénico por estrés térmico, se relacionaron a través de una división los valores del WBGT medio y WBGT límite para cada uno de los puestos de trabajo, obteniendo los resultados que se muestran en la tabla 8. Como se observa en la tabla 8 y en la figura 3, los valores de los índices WBGT medio se encuentran por debajo de los correspondientes a los índices WBGT límite. Por ello se tiene como resultado que no existe riesgo higiénico por estrés térmico, en los puestos de trabajo analizados.

Tabla 8: Evaluación del riesgo por estrés térmico

\begin{tabular}{|c|c|c|c|c|}
\hline $\begin{array}{c}N^{\circ} \\
\text { Puesto } \\
\text { de } \\
\text { trabajo }\end{array}$ & $\begin{array}{c}\text { WBGT medio } \\
\left({ }^{\circ} \mathrm{C}\right)\end{array}$ & $\begin{array}{c}\text { WBGT } \\
\text { LIMITE }\left({ }^{\circ} \mathrm{C}\right)\end{array}$ & Comparativa & Nivel de riesgo \\
\hline 1 & 24,66 & 30 & 0,82 & $\begin{array}{c}\text { NO EXISTE RIESGO } \\
\text { HIGIENICO }\end{array}$ \\
\hline 2 & 24,50 & 30 & 0,82 & $\begin{array}{c}\text { NO EXISTE RIESGO } \\
\text { HIGIENICO }\end{array}$ \\
\hline 3 & 24,43 & 28 & 0,87 & $\begin{array}{c}\text { NO EXISTE RIESGO } \\
\text { HIGIENICO }\end{array}$ \\
\hline 4 & 24,57 & 30 & 0,82 & $\begin{array}{c}\text { NO EXISTE RIESGO } \\
\text { HIGIENICO }\end{array}$ \\
\hline
\end{tabular}

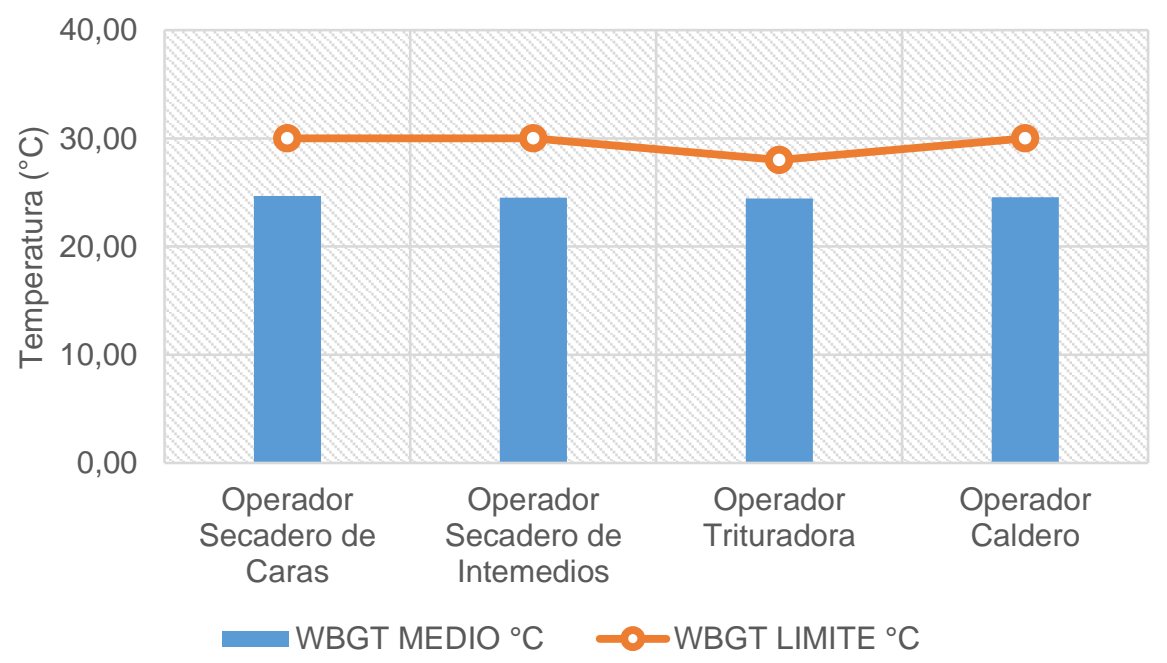

Fig. 3: WBGT Medio y WBGT Límite 
El índice WBGT es el más aceptado y utilizado para medir las variables ambientales sobre el estrés térmico laboral. (Gun y Budd, 1995). Yi y Chan (2015) han revelado que el índice WBGT tiene la mayor validez en la predicción de los impactos debidos a la exposición al calor ocupacional en la industria de la construcción.

Hajizadeh et al. (2016) han evaluado la precisión y validez de varios índices de estrés térmico en hornos de ladrillos en la ciudad de Qom, central Irán. En su estudio se concluye que el índice WBGT, debido a su facilidad de medición y cálculo y al estar aprobado por ISO, es el más aplicable para evaluar estrés térmico en lugares de trabajo. Además se indicó que el índice WBGT puede ser introducido como el índice empírico más válido de estrés por calor en talleres de ladrillos.

En Portugal, Oliveira et al. (2017) han evaluado las condiciones de trabajo en entornos térmicos en la industria del vidrio utilizando el índice WBGT. Obtuvieron como resultado que casi el $80 \%$ de los lugares de trabajo analizados son propensos a condiciones de estrés por calor.

Yang et al. (2017) presentan una evaluación la exposición al calor ocupacional de 12 trabajadores en 5 plantas en un país subtropical. Para ello utilizaron el índice WBGT y las variables del estándar ISO 7933 de acuerdo con la ISO 7243 (2003) e ISO 7933 (2004), respectivamente. Los resultados indicaron que el $42 \%$ de los sujetos (5 trabajadores) superaron los límites del índice WBGT y de acuerdo al tiempo de exposición máximo permitido (ISO 7933) el $42 \%$ de los trabajadores no podían continuar trabajando en ambientes calurosos.

Krishnamurthy et al. (2016), en su estudio realizado en una industria de acero al sur de la India, han demostrado que las altas exposiciones al calor y la gran carga de trabajo afectan la salud de los trabajadores y reducen su capacidad de trabajo. El $90 \%$ de las mediciones del índice WBGT fueron más altas que los valores límite de los umbrales recomendados.

Farshad et al. (2014) han analizado la correlación entre el Índice de Límite de trabajo térmico (TWL), el índice WBGT y el índice de la gravedad específica de la orina (USG) en un sitio de construcción de la ciudad de Teherán, Irán. Las medidas de estos tres índices se relacionaron significativamente, sin embargo el índice TWL presentó algunas ventajas. En cuanto al índice WBGT todas las mediciones estuvieron por debajo del límite y por lo tanto los trabajadores estaban en el nivel permitido de estrés por calor.

\section{Medidas de prevención y control}

La no existencia del riesgo de estrés térmico por exposición a calor en los procesos analizados, no impide la implementación de medidas de prevención y adaptación, tendientes a mejorar los ambientes de trabajo, aumentar el confort de los trabajadores y a evitar que en futuro dicho riesgo se pueda materializar, ya que la creciente exposición al calor debido a cambios climáticos locales puede originar riesgos para la salud y afectar la productividad de los trabajadores (Kjellstrom, 2009), de este modo se proponen las siguientes actividades y medidas de control, cuyo análisis de factibilidad (técnica y económica) e implementación quedará a discreción de la empresa.

Medidas de Control en las Fuentes de Calor: 1) priorizar las acciones de mantenimientos preventivos en caldera, secaderos y otros equipos e instalaciones de trabajo de los procesos, a fin de asegurar su correcto funcionamiento; 2) revisar permanentemente el estado e instalación de los materiales de aislamiento de calor colocados en instalación de transporte de vapor saturado, a fin de evitar pérdidas de energía que a la vez contribuyen al aumento de temperatura en los ambientes de trabajo; y 3) ubicar barreras de material aislante y de absorción de calor en las superficies de las cámaras de secado, a fin de disminuir su nivel de irradiación de temperatura hacia puestos de trabajo cercanos.

Medidas de Control en el Medio de Transmisión: 1) asegurarse que las tuberías de desfogue de condensado y vapor residual se encuentren en los exteriores de las instalaciones, de modo que no contribuyan en el aumento de los niveles de temperatura y humedad al interior del recinto laboral; y 2) garantizar el correcto funcionamiento del sistema de renovación de aire (eólicos de acción mecánica) y de considerarse necesario aumentar su capacidad.

Medidas de Control en los Trabajadores: 1) aunque de acuerdo a los resultados de la evaluación, los trabajos pueden desarrollarse en un régimen continuo durante las 8 horas, implementar pausas de recuperación de al menos 5 minutos por cada dos horas de trabajo; 2) informar y capacitar a los trabajadores sobre los riesgos relacionados con la exposición al calor, los procedimientos de trabajo pertinentes, el uso de equipos de protección personal, y medidas de primeros auxilios; 3) continuar con el suministro de agua potable a lo largo de las instalaciones e incentivar a los trabajadores sobre la importancia de mantener una hidratación permanente; y 4) la empresa al estar ubicada en la Región Amazónica en la ciudad del Puyo, la cual tiene una temperatura promedio de $21,2^{\circ}$, requiere realizar un proceso de inducción para el personal de nuevo ingreso, para lo cual será necesario considerar períodos de aclimatación al menos por una semana, mientras el metabolismo de trabajador se acostumbra al ritmo de trabajo. 


\section{CONCLUSIONES}

Con base en los resultados obtenidos en el proceso de evaluación, se concluye que en ninguno de los puestos de trabajo de los procesos de incineración y secado de la empresa Arboriente S.A. existe riesgo higiénico por estrés térmico, esto se debe principalmente a que su Carga Térmica Metabólica se mantiene entre las 136 y 240 Kilocalorías por hora, niveles en los que según el método ISO 7243 se incluyen los tipos de trabajo "Liviano" (inferior a $200 \mathrm{Kcal} / \mathrm{h}$ ) y "Moderado" (de 200 a $350 \mathrm{Kcal} / \mathrm{h}$ ), también se debe a que los trabajadores pasan por un adecuado proceso de aclimatación previo al inicio de sus labores.

Los valores del WBGT medio de los puestos de trabajo analizados, se encuentran por debajo de los valores límite, por ello y con base en lo establecido en el método ISO 7243 se concluye que es viable mantener un régimen continuo de trabajo durante las 8 horas, sin embargo se recomienda la implementación de pausas de 5 minutos por cada 2 horas de trabajo.

Según Yang et al. (2017), si los valores del índice WBGT superan los valores de referencia, o si se requiere un análisis más detallado, la norma ISO 7933 propone un método analítico para evaluar el estrés térmico. Por lo tanto, es importante mencionar que el presente estudio constituyó el punto de partida en el proceso permanente de evaluación ambiental de calor, como un contaminante de los locales de trabajo, por lo que a más de repetir el proceso periódicamente, es necesario fortalecerlo a través de la aplicación del método de la sobrecargara térmica estimada (ISO 7933:2004), cuya mayor cantidad de variables, especificidad y extensión en sus cálculos, fortalecerá la fiabilidad en los resultados obtenidos (INSHT, 2011).

\section{AGRADECIMIENTOS}

Un agradecimiento especial al Ing. Marcos Vicente Gutiérrez Gualotuña Gerente de Planta de la Empresa Arboriente S.A., quién ha permitido el ingreso a las instalaciones de la empresa para la ejecución de este trabajo.

\section{REFERENCIAS}

Armstrong, L.E.; Y. Epstein.; J.E. Greenleaf.; E.M. Haymes.; R.W. Hubbard.; W.O. Roberts y P.D. Thompson, American College of Sports Medicine position stand. Heat and cold illnesses during distance running, Medicine and Science in Sports and Exercise: 28(12), 1-10 (1996).

Budd, G. M, Wet-bulb globe temperature (WBGT) - its history and its limitations, doi: 10.1016/j.jsams.2007.07.003, Journal of Science and Medicine in Sport: 11(1), 28- 30 (2007).

Camacho, D.I, Estrés Térmico en Trabajadores Expuestos al Área de Fundición en una Empresa Metalmecánica, Mariara. 2004-2005, doi: 10.4067/S0718-24492013000100007, Ciencia \& Trabajo: 15(46), 3-4 (2013).

Cújar, A. D. y G.P. Julio, Evaluación de las condiciones térmicas ambientales del área de producción en una panadería en Cereté (Córdoba), doi: 10.18041/entramado.2016v12n1.23103, Entramado: 12(1), 336-341 (2016).

Curley, J. y S. Irwin., Disorders of temperature control. Part II: Hyperthermia, Irwin and Rippe's Intensive Care Medicine, $5^{\mathrm{a}}$ edición, 66, 762-777, (2003).

Decreto Ejecutivo 2393, Reglamento de Seguridad y Salud de los Trabajadores Mejoramiento del Medio Ambiente de Trabajo, 80-92, Quito, Ecuador (1986).

Dunne, J.P.; R.J. Stouffer y J.G. John., Reductions in labour capacity from heat stress under climate warming, doi: 10.1038/nclimate1827, Nat. Clim. Chang: 3, 563-566 (2013).

Epstein, Y. y D.S. Moran., Thermal Comfort and the Heat Stress Indices, doi: 10.2486/indhealth.44.388, Industrial Health: 44(3), 388-398 (2006).

Falagán, M.J.; A. Canga.; P. Ferrer y J.M. Fernández, Manual Básico de Prevención de Riesgos Laborales: Higiene industrial, Seguridad y Ergonomía, 1a edición, 25-26. Sociedad Asturiana de Medicina y Seguridad en el Trabajo y Fundación Médicos Asturias, Oviedo, España (2000).

Farshad, A.; S. Montazer.; M. R. Monazzam.; M. Eyvazlou y R. Mirkazemi., Heat stress level among construction workers. Iran J Public Health: 43(4), 492-498 (2014).

Fisk, W.J, Health and productivity gains from better indoor environments and their relationship with building energy efficiency, doi: 10.1146/annurev.energy.25.1.537, Annual Review of Energy and the Environment: 25(1), 537-566 (2000).

Gun, R.T. y G.M. Budd., Effects of thermal, personal and behavioural factors on the physiological strain, thermal comfort and productivity of Australian shearers in hot weather, doi: 10.1080/00140139508925195, Ergonomics: 38(7), 1368-1384 (1995).

Hajizadeh, R.; F. Golbabaei.; S. Farhang Dehghan.; M.H. Beheshti.; S.M. Jafari y F. Taheri., Validating the Heat Stress Indices for Using In Heavy Work Activities in Hot and Dry Climates, J Res Health Sci: 16(2), 90-95 (2016). 
Hernández, R.; C. Fernández y P. Baptista, Metodología de la Investigación, 6a edición, 188-192. McGraw-Hill, México D.F, México (2014).

INHAMI, Anuario Meteorológico No ${ }^{\circ} 52-2012$, (en la web: http://www.serviciometeorologico.gob.ec/wpcontent/uploads/anuarios/meteorologicos/Am\%202012.pdf, acceso: 12 de junio 2017), Naranjo, C., Quito- Ecuador (2015).

INSHT NTP 322, Instituto Nacional de Seguridad e Higiene en el Trabajo, Valoración del riesgo de estrés térmico: índice WBGT, 1-6, España (1993).

INSHT NTP 923, Instituto Nacional de Seguridad e Higiene en el Trabajo, Estrés térmico y sobrecarga térmica: evaluación de los riesgos (II), 1-7, España (2011).

ISO 7243, International Organization for Standardization (ISO), Hot environments - Estimations of the heat stress on working man, based on the WBGT- index (Wet Bulb Globe Temperature), 1-9, Geneva- Switzerland (1989).

Kjellstrom, T.; H. Ingvar y B. Lemke, Workplace heat stress, health and productivity - an increasing challenge for low and middle-income countries during climate change, Global Health Action: 2(1), 1-6 (2009).

Kjellstrom, T.; D. Briggs.; C. Freyberg.; B. Lemke.; M. Otto y O. Hyatt., Heat, human performance, and occupational health: A key issue for the assessment of global climate change impacts, doi: 10.1146/annurev-publhealth-032315-021740, Annu Rev Public Health: 37, 97-112 (2016).

Kjellstrom, T.; C. Freyberg.; B. Lemke.; M. Otto y D. Briggs., Estimating population heat exposure and impacts on working people in conjunction with climate change, doi: 10.1007/s00484-017-1407-0, Int. J. Biometeorol. 1-16 (2017).

Krishnamurthy, M.; P. Ramalingam y otros 5 autores., Occupational heat stress impacts on health and productivity in a steel industry in southern India, doi: 10.1016/j.shaw.2016.08.005, Saf Health Work: 8(1), 99-104 (2017).

Lundgren, K.; K. Kuklane.; C. Gao y I. Holmér., Effects of heat stress on working populations when facing climate change, Industrial Health, 51(1), 3-15 (2013).

MMWR. Heat-related deaths among crop workers_United States, 1992_2006. JAMA: 300, 1017- 1018 (2008).

Montain, S.J.; W.A. Latzka y M.N. Sawka, Fluid replacement recommendations for training in hot weather, Military Medicine: 164 (7), 502-508 (1999).

Moran, D.S. y K.B. Pandolf, Wet bulb globe temperature (WBGT)--to what extent is GT essential?, Aviation, Space, and Enviromental Medicine: 70 (5), 480-484 (1999).

Oliveira, A. V. M.; A. R. Gaspar.; A.M. Raimundo y D.A. Quintela., Assessment of thermal environments: Working conditions in the Portuguese ceramic industry in 1994 and 2012, doi: 10.2486/indhealth.2017-0048, Work, 51(3), 457-470 (2015).

Parsons, K, Heat Stress Standard ISO 7243 and its Global Application, doi: 10.2486/indhealth.44.368, Industrial Health: 44(3), 368-372 (2006).

Pérez, A.; B. Fernández y A.M. Álvarez., El golpe de calor, doi: 10.1016/S1134-2072(04)76122-8, FMC - Formación Médica Continuada en Atención Primaria: 11(6), 287-292 (2004).

Piñeiro, N.; J.L. Martínez.; E. Alemparte y J.C. Rodríguez., Golpe de calor, Emergencias: 16, 116-125 (2004).

Ramsey, J.D, Abbreviated guidelines for heat stress exposure, doi: 10.1080/0002889778507794, American Industrial Hygiene Association Journal: 39(6), 491-495 (1978).

Tawatsupa, B.; L.Y. Lim.; T. Kjellstrom.; S. Seubsman y A. Sleigh., The association between overall health, psychological distress, and occupational heat stress among a large national cohort of 40,913 Thai workers, doi: 10.3402/gha.v3i0.5034, Global Health Action: 3(1), 1-10 (2010).

Tawatsupa, B.; V. Yiengprugsawan.; T. Kjellstrom.; J Berecki-Gisolf.; S.A. Seubsman y A. Sleigh., Association between heat stress and occupational injury among Thai workers: findings of the Thai Cohort Study, doi: 10.2486/indhealth.20120138, Industrial health, 51(1), 34-46 (2013).

Vandentorren, S.; P. Bretin y otros ocho autores., August 2003 heat wave in France: risk factors for death of elderly people living at home, doi: 10.1093/eurpub/ckl063, Eur J Public Health: 16(6), 583-591 (2006).

Wyndham, C.H, A survey of the causal factors in heat stroke and of their prevention in the gold mining industry, JournalSouth African Institute of Mining and Metallurgy: 66(4), 125-155 (1965).

Wyon, D.P, The effects of indoor air quality on performance and productivity, doi: 10.1111/j.1600-0668.2004.00278.x, Indoor Air: 14(7), 92-101 (2004).

Yang, Y. C.; M.C. Wei y S.J. Hong., Evaluation of occupation hot exposure in industrial workplaces in a subtropical country, doi: 10.13075/ijomeh.1896.00761, Int J Occup Med Environ Health, 30(3), 379-395 (2017).

Yi, W. y A.P. Chan., Effects of temperature on mortality in Hong Kong: a time series analysis, doi: 10.1007/s00484-0140895-4, Int J Biometeorol: 59(7), 927-936 (2015).

Yi, W. y A.P. Chan., Effects of Heat Stress on Construction Labor Productivity in Hong Kong: A Case Study of Rebar Workers, doi: 10.3390/ijerph14091055, Int J Environ Res Public Health: 14(9), 1-14 (2017). 
